\title{
STUDIES ON THE IONOPHOROUS ANTIBIOTICS \\ VIII. EFFECTS OF MONOVALENT AND DIVALENT CATION IONOPHORES ON BLOOD PLATELETS
}

\author{
Mitsuaki Mitani, Teruhiko Umetsu and Tadashi Yamanishi \\ Research Laboratory, Kaken Chemical Co., Ltd. \\ Bunkyo-ku, Tokyo 113, Japan \\ NOBORU ŌTAKE \\ Institute of Applied Microbiology, University of Tokyo \\ Bunkyo-ku, Tokyo 113, Japan
}

(Received for publication December 23, 1976)

\begin{abstract}
The effects of a monovalent cation ionophore etheromycin (CP-38295) and a divalent cation ionophore lysocellin on blood platelets function have been investigated. When platelets were incubated with $3 \mu \mathrm{M}$ of each ionophore, the simultaneous induction of secretion of stored serotonin and efflux of intracellular calcium occurred, but there was no detectable influx of extracellular calcium. With increasing concentrations, these compounds caused platelet aggregation accompanied by increased influx of external calcium. These results are consistent with the hypothesis that the liberation of calcium from the intracellular store mediates the platelet secretion reaction.
\end{abstract}

Etheromycin (C20-12) is a new monocarboxylic polyether antibiotic which is crystallized as the sodium salt from the culture broth of Streptomyces hygroscopicus and is identical with the compound CP-38295 (Pfizer). ${ }^{1,2,3)}$ Lysocellin is a monocarboxylic antibiotic produced by Streptomyces cacaoi whose structural elucidation is established by three-dimensional X-ray analysis." Lysocellin is a broad cation selective ionophore which is able to transport monovalent and divalent metal cations as well as biogenic amines across a carbon tetrachloride barrier layer. ${ }^{5 !}$

It has been postulated that the excitation-secretion coupling processes of intracellular calcium regulate general cellular function by interacting with cyclic nucleotides. ${ }^{6,7)}$ The divalent cation ionophores lasalocid A and A23187, which render membranes permeable to calcium, have recently served as valuable tools for understanding molecular mechanism of cell activation systems. ${ }^{8,9,10)}$ In human platelets, these antibiotics stimulate calcium uptake and/or induce redistribution of intracellular calcium, thereby inducing the secretion of serotonin and adenine nucleotides as well as aggregation. ${ }^{11,121}$

In this paper, studying the effects of etheromycin and lysocellin, ionophores of characteristic cation selectivity, on calcium movement and serotonin secretion in rabbit blood platelets, we demonstrate results which provide some support for a hypothesis that the platelet secretion reaction might be triggered by the cytoplasmic calcium released from the intracellular pools.

\section{Materials and Methods}

Etheromycin and lysocellin used in the present study were stock samples in our laboratory. Lasalocid A was a generous gift from Dr. T. WiLliams of Hoffmann-La Roche, Nutley, New Jersey, U.S.A. ${ }^{22} \mathrm{Na},{ }^{86} \mathrm{Rb},{ }^{45} \mathrm{Ca},{ }^{3} \mathrm{H}$-serotonin, ${ }^{3} \mathrm{H}$-norepinephrine and ${ }^{14} \mathrm{C}$-histamine were purchased from the Radiochemical Centre, Amersham, England and ${ }^{42} \mathrm{~K}$ from Japan Atomic Energy Research Institute, Tokyo, Japan. 
The association constants of the antibiotics for various cations or amines were determined in two-phase distribution experiments on the assumptions of $1: 1$ complexes with monovalent cations and amines and 2:1 complexes with divalent cations as described in the previous report. ${ }^{5)}$

Platelets were isolated by centrifuging the blood collected from normal male rabbits and washed twice, once with saline containing $1 \mathrm{~mm}$ EDTA and $25 \mathrm{~mm}$ Tris-maleate, $\mathrm{pH} 6.8$ and once with saline buffered by $25 \mathrm{~mm}$ Tris-maleate, $\mathrm{pH} 6.8$ and finally suspended in saline at a concentration of $2 \times 10^{9}$ platelets per $\mathrm{ml}$.

Platelets preloaded with ${ }^{3} \mathrm{H}$-serotonin were prepared by incubating the platelets with ${ }^{3} \mathrm{H}$-serotonin at $25^{\circ} \mathrm{C}$ for 60 minutes. The uptake of ${ }^{45} \mathrm{Ca}$ was studied with ${ }^{3} \mathrm{H}$-serotonin preloaded platelets as follows: after 2-minute incubation with various concentrations of ionophores at $37^{\circ} \mathrm{C}$, the reaction was terminated by addition of chilled saline and followed by immediate centrifugation. Aliquots of the supernatant fluid were used for counting the radioactivity of ${ }^{3} \mathrm{H}$-serotonin. The precipitated platelets were washed once with saline and finally resuspended in water. After addition of 0.5 volume of $0.3 \mathrm{~N}$ $\mathrm{KOH}$, aliquots were used for measurement of the radioactivity of ${ }^{45} \mathrm{Ca}$. Thus both ${ }^{45} \mathrm{Ca}$ and ${ }^{3} \mathrm{H}$-serotonin were measured in the same experiment.

In the calcium release experiment, platelets previously loaded with ${ }^{45} \mathrm{Ca}$ at $37^{\circ} \mathrm{C}$ for 90 minutes were incubated with ionophores at $37^{\circ} \mathrm{C}$ for 5 minutes and the reaction was terminated by immediate centrifugation. The amount of ${ }^{45} \mathrm{Ca}$ released from platelets into the supernatant fluid was measured. All samples were counted in dual channel liquid scintillation spectrometer and the counts of ${ }^{3} \mathrm{H}$-channel were corrected for spill-over of ${ }^{45} \mathrm{Ca}$.

Platelet aggregation was measured as a change of transmitted light with an aggreometer.

\section{Results}

In a two-phase distribution study, etheromycin exhibited a greater binding affinity for $\mathrm{K}^{+}$than the other organic or inorganic cations (Table 1). In contrast, lysocellin displayed a broad range cation selectivity. The antibiotic showed preferences not only for monovalent and divalent metal cations but also for biogenic amines (Table 1). These results show that etheromycin is a monovalent cation ionophore and lysocellin, on the other hand, is an ionophore with a broad-range cation selectivity profile.

When platelets were incubated with $3 \mu \mathrm{m}$ etheromycin, almost complete secretion of ${ }^{3} \mathrm{H}$-serotonin

Fig. 1. Serotonin secretion and calcium uptake induced by various ionophores in platelets. Platelets preloaded with ${ }^{3} \mathrm{H}$-serotonin (final concentration, $10^{9}$ per $\mathrm{ml}$ ) were incubated with ionophores in a medium containing $25 \mathrm{~mm}$ Tris- $\mathrm{HCl}, \mathrm{pH} 7.4 ; 130 \mathrm{~mm} \mathrm{NaCl} ; 5 \mathrm{~mm} \mathrm{KCl} ; 5 \mathrm{~mm}$ glucose; and $0.2 \mathrm{~mm}$ ${ }^{45} \mathrm{Ca}(0.2 \mu \mathrm{Ci})$. Final volume, $1 \mathrm{ml}$; temperature, $37^{\circ} \mathrm{C}$. See "Materials and Methods" for experimental details.

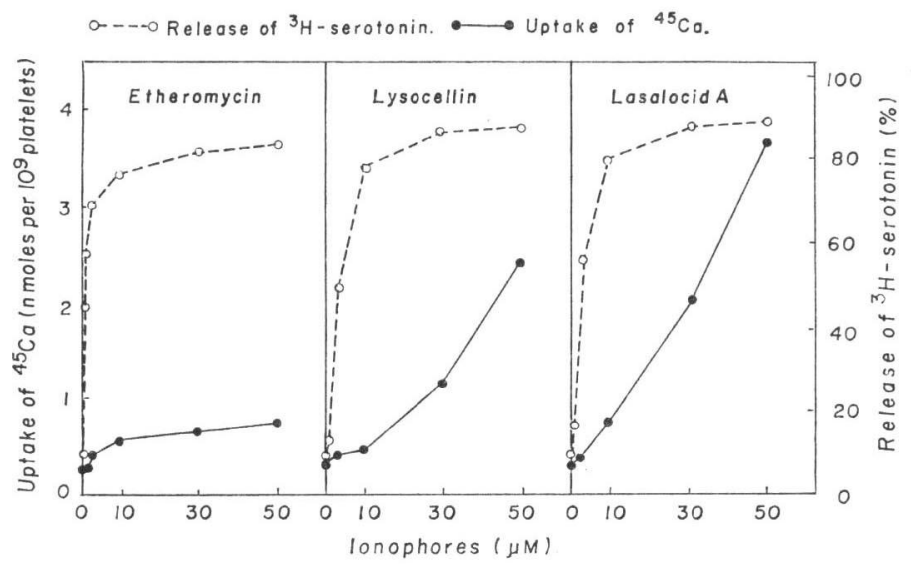


stored in platelets occurred without detectable influx of externally added ${ }^{45} \mathrm{Ca}$ (Fig. 1). With increasing etheromycin concentrations, a small and limited amount of ${ }^{45} \mathrm{Ca}$ was taken up by platelets. The addition of $3 \mu \mathrm{M}$ lysocellin also induced complete serotonin release, but did not produce significant influx of ${ }^{45} \mathrm{Ca}$ (Fig. 1). With $30 \mu \mathrm{M}$ lysocellin, however, a great increase in ${ }^{45} \mathrm{Ca}$ influx was observed. Another calcium ionophore lasalocid A displayed the similar influences on serotonin secretion and calcium uptake (Fig. 1). These results suggest that the influx of external calcium into platelets may not be necessary for triggering serotonin secretion. To learn more about this possibility, we studied the effect of ionophores stimulation on the release of calcium from the platelets preloaded with ${ }^{45} \mathrm{Ca}$. As shown in Fig. 2, the addition of $0.1 \mu \mathrm{M}$ etheromycin or lysocellin caused an increased efflux of ${ }^{45} \mathrm{Ca}$ from platelets. It seems likely in this case that (1) the ionophores probably mobilize calcium from the accumulating organelles into the cytoplasm, thereby increasing cytoplasmic calcium concentration, (2) since the ionophores render membranes permeable to the cation, these increased levels of cytoplasmic $\mathrm{Ca}^{2+}$ resulted in an enhancement of $\mathrm{Ca}^{2+}$ efflux. It therefore seems very likely that the serotonin secretion reaction is mediated by the intracellular release of calcium.

Fig. 3 shows the time course of platelet aggregation induced by ionophores. With the high concentrations of $30 \mu \mathrm{M}$, coarse and dense aggregates of platelets were produced by both

Table 1. $\mathrm{K}_{\mathrm{A}}$ of etheromycin and lysocellin for various metal cations and amines

\begin{tabular}{l|c|c}
\hline \multicolumn{1}{c}{ Cation or amine } & Etheromycin & Lysocellin \\
\hline $\mathrm{Na}^{+}$ & 0.46 & 0.21 \\
$\mathrm{~K}^{+}$ & 0.85 & 0.51 \\
$\mathrm{Rb}^{+}$ & 0.32 & 0.52 \\
$\mathrm{Mg}^{2+}$ & 0.1 & 50.8 \\
$\mathrm{Ca}^{2+}$ & 0.1 & 49.2 \\
Serotonin & 0.13 & 4.56 \\
Norepinephrine & 0.007 & 0.13 \\
Histamine & 0.007 & 0.31 \\
\hline
\end{tabular}

The compounds dissolved in $0.1 \mathrm{ml}$ of $25 \%$ dimethylformamide plus $75 \%$ ethanol were mixed vigorously with $1 \mathrm{ml}$ of a $30 \% n$-butanol-70\% toluene mixture and $0.5 \mathrm{ml}$ of aqueous buffer containing $0.1 \sim 20 \mathrm{~mm}$ radioactive metal salts or amines and $40 \mathrm{~mm}$ Tricine buffer, $\mathrm{pH} 8.3$. The antibiotics were used at a concentration of $5 \times 10^{-4} \mathrm{M}$ and the values of association constant $\left(\mathrm{K}_{\mathrm{A}}\right)$ were calculated from the following equations:

for monovalent cation and amine,

$$
\mathrm{K}_{\mathrm{A}}=\frac{\left[\text { Ionophore }^{-} \cdot \text { Cation }_{\mathrm{org}}^{+}\right]}{\left[\text {Ionophore }_{\mathrm{org}}^{-}\right] \cdot\left[\text { Cation }_{\mathrm{H}_{2} \mathrm{O}}^{+}\right]}
$$

for divalent cation,

$$
\mathrm{K}_{\mathrm{A}}=\frac{\left[\text { Ionophore }_{2}^{-} \cdot \text { Cation }_{\mathrm{org}}^{2+}\right]}{\left[\text { Ionophore }_{\mathrm{org}}^{-}\right]^{2} \cdot\left[\text { Cation }_{\mathrm{H}_{2} \mathrm{O}}^{2+}\right]}
$$

For $\mathrm{K}_{\mathrm{A}}$ multiply above values by $10^{3}$.

Fig. 2. Ionophore-induced efflux of ${ }^{45} \mathrm{Ca}$ from platelets.

Platelets preloaded with ${ }^{45} \mathrm{Ca}$ were incubated with ionophores in a medium containing $25 \mathrm{~mm}$ Tris$\mathrm{HCl}, \mathrm{pH} 7.4 ; 130 \mathrm{~mm} \mathrm{NaCl}$; and $5 \times 10^{8}$ platelets. Final volume, $1 \mathrm{ml}$; temperature, $37^{\circ} \mathrm{C}$. See "Materials and Methods" for details.

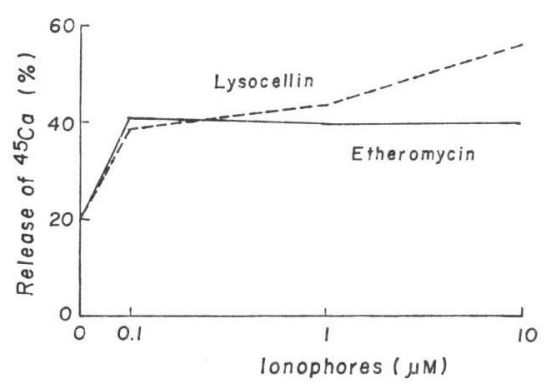

Fig. 3. The time course of platelet aggregation induced by the ionophores.

The reaction system contained $25 \mathrm{~mm}$ Tris- $\mathrm{HCl}$, pH 7.4; $130 \mathrm{~mm} \mathrm{NaCl} ; 5 \mathrm{~mm} \mathrm{KCl} ; 5 \mathrm{~mm}$ glucose; $0.2 \mathrm{mM} \mathrm{CaCl}_{2}$; and $2 \times 10^{8}$ platelets in a final volume of $1 \mathrm{ml}$. Temperature, $37^{\circ} \mathrm{C}$. Antibiotic additions at indicated points.

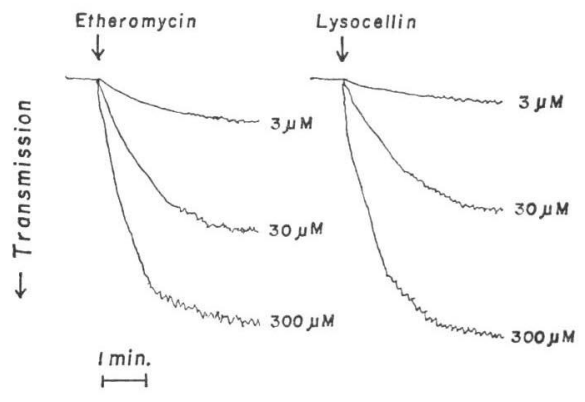


compounds. These results indicate that the platelet aggregation may be associated with the processes leading to a simultaneous induction of serotonin release and calcium influx.

\section{Discussion}

The complexation affinity determined by two-phase distribution techniques shows that etheromycin is a monovalent cation ionophore which exhibits more preferences for alkali metal cations than for divalent cations or amines (Table 1). Lysocellin, in contrast, displayed a broad range cation selectivity (Table 1). It is noteworthy that lysocellin exhibited a high binding affinity for biogenic amines such as serotonin, 1-norepinephrine and histamine. With respect to the cation selectivity, the compound resembles lasalocid A. $^{10)}$

When platelets were incubated with $3 \mu \mathrm{M}$ of each ionophore, complete secretion of stored serotonin was induced by etheromycin and lysocellin as well as lasalocid A, but there was no detectable influx of extracellular $\mathrm{Ca}^{2+}$ (Fig. 1). With increasing concentrations, these compounds caused platelet aggregation accompanied by an increased calcium influx (Figs. 1 and 3). These results indicate that platelet secretion reaction may be mediated by the internal calcium. This is in agreement with the facts reported by MüRER et al. ${ }^{12)}$ that the calcium ionophore A23187 causes massive release of stored serotonin accompanied by intracellular accumulation of inosine monophosphate, but produces no detectable influx of externally added calcium. The possible role of internal calcium in the release of serotonin or ATP has also been reported by FeINMAN and DETWILER. ${ }^{11,13)}$

In ${ }^{45} \mathrm{Ca}$ release experiments, we found that etheromycin and lysocellin caused an increased efflux of ${ }^{45} \mathrm{Ca}$ from platelets which was thought to reflect the mobilization of calcium from the storing organelles (Fig. 2). The similar increase in calcium efflux by platelets upon thrombin stimulation has been demonstrated by MASSINI et al. ${ }^{14)}$ Recently, with the use of chlortetracycline fluorescent probe, LE BRETON et al. ${ }^{15)}$ have provided direct evidence for the intracellular calcium redistribution associated with the shape change of platelets.

Our findings that a monovalent cation ionophore etheromycin causes massive release of serotonin and calcium from platelets indicate the complexity of the mode of action of this compound. Hitherto, there are only few reports which show the effects of monovalent cation ionophores on calcium distribution in biological systems. ROTTENBERG and SCARPA ${ }^{16}$ have reported that nigericin increased the rate and extent of $\mathrm{Ca}^{2+}$ uptake by rat liver mitochondria at low concentrations but caused release of calcium at high concentrations. PRESSMAN and DEGUZMAN ${ }^{101}$ have reported the inotropic effects of monovalent cation ionophores such as monensin, dianemycin, X-206 and A-204. Recently, LowE et $a l .{ }^{17)}$ proposed the possibility that the intracellular sodium acts as "a second messenger" in exocytotic release processes in the pancreatic $\beta$ cell by releasing calcium from the intracellular pools. This possibility together with the observation ${ }^{181}$ that potassium and sodium have opposite effects on the uptake of calcium by platelets, the former increasing and the latter decreasing uptake, are thought to provide valuable clues for understanding the mode of action of etheromycin. The preliminary results with rat liver mitochondria showed that both etheromycin and lysocellin caused the simultaneous release of monovalent and divalent metal cations from mitochondria. These results will be published in the following report.

\section{Acknowledgements}

The authors wish to thank Prof. Hiroshi YoneHARA of Institute of Applied Microbiology, University of Tokyo and Dr. Yukio Inoue of Kaken Chemical Co., Ltd. for their keen discussions. This work was supported in part by a grant from the Japanese Ministry of Education, Science and Culture.

\section{References}

1) Kusakabe, Y.; C. Nagatsu, H. Akutsu, C. Hirose, M. Miyamoto \& A. Seino: The studies on a new antibiotic C20-12. Abstracts papers of Annual Meeting of the Agricultural Chemical Society, Japan. 
p. 83, Sapporo, 1975

2) Celmer, W. D.; W. P. Cullen, M. T. Jefferson, J. B. Routien, F. C. Sciavolino \& C. E. Moppett: Als Verbindung 38295 bezeichnetes Antibiotikum, Verfahren zu seiner Herstellung und seine Verwendung. Deutsch. Offen. 2534342, Feb. 19, 1976

3) Cullen, W. P.; L. R. Chappel, M. T. Jefferson, C. E. Moppett, J. B. Routien, F. C. Sciavolino \& W. D. Celmer: CP-38,295, a new polycyclic ether antibiotic. 16th Interscience Conference on Antimicrobial Agents and Chemotherapy. Paper 234, Chicago, October 27 29, 1976

4) Ōtake, N.; M. Koenuma, H. Kinashi, S. Sato \& Y. Saito: The crystal and molecular structure of the silver salt of lysocellin, a new polyether antibiotic. J.C.S. Chem. Comm. 1975: 92 93, 1975

5) Mitani, M.; T. Yamanishi, E. Ebata, N. Ōtake \& M. Koenuma: Studies on the ionophorous antibiotics. VII. A broad cation selective ionophore, lysocellin. J. Antibiotics 30: 186 188, 1977

6) Rasmussen, H.; P. Jensen, W. Lake, N. Friedmann \& D. B. P. Goodman: Cyclic nucleotides and cellular calcium metabolism. In Advances in Cyclic Nucleotide Research. Vol. 5 ed. by G. I. DRUMmOND, P. Greengard and G. A. Robison. Raven Press, New York, pp. 375 394, 1975

7) Foreman, J. C.; J. L. Mongar \& B. D. Gomperts: Calcium ionophores and movement of calcium ions following the physiological stimulus to a secretory process. Nature 245: 249 251, 1973

8) Maino, V. C.; N. M. Green \& M. J. Crumpton: The role of divalent cations in the initiation of lymphocyte activation. In Immune Recognition. ed. by A. S. Rosenthal. Academic Press, New York, pp. $417 \sim 443,1975$

9) Karl, R. C.; W. S. Zawalich, J. A. Ferrendelli \& F. M. Matschinsky: The role of $\mathrm{Ca}^{2+}$ and cyclic and cyclic adenosine $3^{\prime}: 5^{\prime}$-monophosphate in insulin release induced in vitro by the divalent cation ionophore A23187. J. Biol. Chem. 250: 4575 4579, 1975

10) Pressman, B. C. \& N. T. DeGuzman: Biological application of ionophores: Theory and practice. Ann. New York Acad. Sci. 264: 373 393, 1975

11) Feinman, R. D. \& T. C. Detwiler: Platelet secretion induced by divalent cation ionophores. Nature 249: $172 \sim 173,1974$

12) Mürer, E. H.; G. J. Stewart, M. A. Rausch \& H. J. Day: Calcium ionophore A23187 (Eli Lilly). Effect on platelet function, structure and metabolism. Thrombos. Diathes. Haemorrh. (Stuttg.) 34: $72 \sim 82,1975$

13) Detwiler, T. C. \& R. D. Feinman: Kinetics of the thrombin-induced release of adenosine triphosphate by platelets. Comparison with release of calcium. Biochemistry 12: 2462 2468, 1973

14) MAssini, P. \& E. F. LÜSCHER: On the significance of the influx of calcium ions into stimulated human blood platelets. Biochim. Biophys. Acta $436: 652 \sim 663,1976$

15) Le Breton, G. C.; R. J. Dinerstein, L. J. Roth \& H. Feinberg: Direct evidence for intracellular divalent cation redistribution associated with platelet shape change. Biochem. Biophys. Res. Commun. 71: 362 370,1976

16) Rottenberg, H. \& A. Scarpa: Calcium uptake and membrane potential in mitochondria. Biochemistry 13: $4811 \sim 4817,1974$

17) Lowe, D. A.; B. P. Richardson, P. TAYlor \& P. Donatsch: Increasing intracellular sodium triggers calcium release from bound pools. Nature 260: 337 338, 1976

18) Steiner, M. \& T. TAteishi: Distribution and transport of calcium in human platelets. Biochim. Biophys. Acta 367: $232 \sim 246,1974$ 